\title{
METASnake: a Snakemake workflow to facilitate automated processing of metagenomic data through the metaWRAP pipeline [version 1; peer review: 1 approved]
} John KrapohI(D), Brett E. Pickett (i)

Department of Microbiology and Molecular Biology, Brigham Young University, Provo, UT, 84602, USA

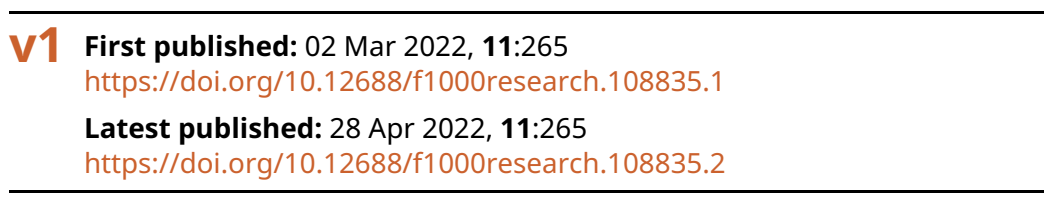

\section{Abstract}

Generating high-quality genome assemblies of complex microbial populations from shotgun metagenomics data is often a manually intensive task involving many computational steps. METASnake is a novel tool, implemented in the Snakemake workflow language, to automate multiple metaWRAP modules. Specifically, it wraps the shell scripts provided within the original metaWRAP software, within Snakemake. This approach enables high-throughput simultaneous assembly and analysis of multiple shotgun metagenomic datasets using the robust modular metaWRAP software. We expect this advancement to be of import in institutions where high-performance computing infrastructure is available, especially in the context of big data. This software tool is publicly available at https://github.com/jkrapohl/METASnake.

\section{Keywords}

metaWRAP, shotgun metagenomics, high-performance computing, big data, Snakemake

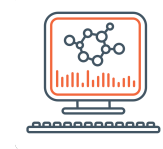

This article is included in the Bioinformatics gateway.

\author{
Open Peer Review \\ Approval Status \\ 1

\section{version 2} \\ (revision) \\ 28 Apr 2022 \\ version 1 \\ 02 Mar 2022

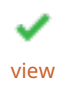 \\ 1. Tobias Jakobi ID, University of Arizona, \\ Phoenix, USA \\ Any reports and responses or comments on the \\ article can be found at the end of the article.
}


Corresponding author: Brett E. Pickett (brett_pickett@byu.edu)

Author roles: Krapohl J: Software, Writing - Original Draft Preparation; Pickett BE: Supervision, Writing - Review \& Editing

Competing interests: No competing interests were disclosed.

Grant information: The author(s) declared that no grants were involved in supporting this work.

Copyright: $\odot 2022$ Krapohl J and Pickett BE. This is an open access article distributed under the terms of the Creative Commons Attribution License, which permits unrestricted use, distribution, and reproduction in any medium, provided the original work is properly cited.

How to cite this article: Krapohl J and Pickett BE. METASnake: a Snakemake workflow to facilitate automated processing of metagenomic data through the metaWRAP pipeline [version 1; peer review: 1 approved] F1000Research 2022, 11:265

https://doi.org/10.12688/f1000research.108835.1

First published: 02 Mar 2022, 11:265 https://doi.org/10.12688/f1000research.108835.1 


\section{Introduction}

As sequencing technology has become cheaper and more readily accessible, the need for the increased computational capacity to process these data has become apparent. In particular, high-throughput sequencing has been particularly useful when applied to the field of metagenomics. Substantial effort has been devoted to developing software and computational pipelines, such as MetaWRAP, which cater to this growing area of research. MetaWRAP combines many of the necessary tools to process reads, create bins, and visualize data within a robust modular design (Uritskiy, DiRuggiero, \& Taylor, 2018). The primary limitation arising from this design is the inability to automatically scale its usage to massive datasets. Snakemake is a widely-used Python based workflow management system that automates repetitive tasks, allowing software processes to be both scalable and reproducible (Mölder et al., 2021). By integrating the original MetaWRAP processes into Snakemake, the customizable modular nature of MetaWRAP can be preserved even when automatically processing large datasets through a single workflow. Our METASnake software automates the tasks performed within MetaWRAP, allowing for individual modules to be toggled on and off using Snakemake-defined "rules".

\section{Methods}

Implementation

As this tool makes use of Snakemake, individual steps of the workflow are broken into rules, many of which can be toggled (Mölder et al., 2021). To do this, Snakemake requires a yaml configuration file as an input to determine which steps are specified for the run, as well detailed parameters such as which assembly tool to use. This configuration file also specifies the location of a metadata file, which contains a list of files associated with the run. An example of both the yaml and metadata file are included in the Github repository. METASnake can be submitted for scheduling from the command line by using the submission script also found within the repository. Running the script requires the complete installation of both Snakemake and METAWrap, as well as all of their dependencies. It is recommended that Snakemake and METAWrap be installed using the latest version of Miniconda, following instructions found on their corresponding Github repositories (Mölder et al., 2021; Orjuela, Huang, Hembach, Robinson, \& Soneson, 2019).

\section{Operation}

The advantage of using METASnake over the base METAWrap program is the ability to appropriately support largescale job sizes while requiring less user input by automating the entire process. This enables jobs to be better organized and allows improved consistency in outputs. We recommended that this tool be run on cluster computing or within a highperformance infrastructure for larger jobs. In such cases, we have found that a minimum of 100 GB of RAM across 24 CPUs is sufficient, with larger jobs requiring additional resources.

The rules found within the Snakemake file correspond to different steps within the original METAWrap software. Some initial required steps include read quality control, assembly, binning, and bin refinement and reassembly. However, we have provided toggle settings for memory-heavy modules that generate figures, which are Blobology, Kraken, bin quantification, bin annotation, and bin classification. This design reduces resource usage by only generating the desired figures, and allows the modification of this setting to create these figures at a later time by toggling the module on in the yaml file, deleting any outputs created for that module, and resubmitting the job. Snakemake will skip any rules already completed and only run the required rule(s).

\section{Use cases}

As stated above, this tool works best with a high-performance computing infrastructure. We anticipate that the most effective use of this tool is applying it to big metagenomics data, such as meta-analyses of existing datasets or datasets with many samples. To assess the capabilities of this tool, the publicly available samples SRR13296364 and SRR13296365, accessed from the NCBI Sequence Read Archive from the study SRP299130, were run together successfully. We then ran a larger set of files from the SRP257563 study through the metaWRAP pipeline using this software. We found that this tool will run as expected as long as sufficient memory is provided and all dependencies are installed correctly. Inputs include sequencing files in fastq format, a metadata file listing all fastq filenames, and a yaml file describing paths for all file sources and destinations. Outputs include high-quality genomic bins, as wells as a number of generated figures such as a blobplot, heatmap, and kronogram.

\section{Discussion}

While the processing of metagenomics datasets is untractable for most personal computers, researchers with access to high-performance computing infrastructure can take full advantage of this software. The core functions found within the original MetaWRAP include read quality control, assembly, and binning are required for the analysis and cannot be toggled off. These functions include several refinement steps that are unique to MetaWRAP, which allow it to create higher-quality bins than existing stand-alone programs (Uritskiy et al., 2018). Our design enables the user to decide 
whether computationally-expensive modules, such as Kraken and Blobology, that assist with figure generation can be skipped.

Snakemake automatically generates a directed acyclic graph (DAG) to order tasks, track the progress of each task for each sample, and eliminate duplicate tasks for the same sample (Mölder et al., 2021). This is vital to efficiently processing and analyzing large datasets, as jobs can fail due to insufficient memory or timing out. An advantage of implementing this workflow in Snakemake is the ability to resume the workflow from the same step that was underway if a process fails. The input paths, output paths, and parameters for each job are assigned by the end-user within a configuration file. This file is read by Snakemake to prevent data from being incorrectly assigned or lost and to facilitate reuse and customization of the workflow. We anticipate that this advance in automating metagenomics data processing will facilitate the generation, analysis, and re-analysis of larger datasets in the future.

\section{Data availability}

No data are associated with this article.

\section{Software availability}

Source code available from: https://github.com/jkrapohl/METASnake.

Archived DOI at time of publication: https://doi.org/10.5281/zenodo.5719960.

License: Open.

\section{Acknowledgements}

We would like to thank the Brigham Young University (BYU) Office of Research Computing as well as BYU for providing the facilities for this work.

Mölder F, Jablonski KP, Letcher B, et al.: Sustainable data analysis with Snakemake. F1000Res. 2021; 10: 33 .

PubMed Abstract | Publisher Full Text

Orjuela S, Huang R, Hembach KM, et al.: ARMOR: An Automated Reproducible MOdular Workflow for Preprocessing and Differential
Analysis of RNA-seq Data. G3 (Bethesda). 2019; 9(7): 2089-2096. PubMed Abstract | Publisher Full Text

Uritskiy GV, DiRuggiero J, Taylor J: MetaWRAP-a flexible pipeline for genome-resolved metagenomic data analysis. Microbiome. 2018; 6(1): 158.

PubMed Abstract | Publisher Full Text 


\section{Open Peer Review}

\section{Current Peer Review Status:}

\section{Version 1}

Reviewer Report 29 March 2022

https://doi.org/10.5256/f1000research.120268.r126391

(C) 2022 Jakobi T. This is an open access peer review report distributed under the terms of the Creative Commons Attribution License, which permits unrestricted use, distribution, and reproduction in any medium, provided the original work is properly cited.

\section{Tobias Jakobi}

Department of Internal Medicine and the Center for Translational Cardiovascular Research, University of Arizona, Phoenix, AZ, USA

The manuscript submitted by Krapohl et al. outlines a Snakemake-based framework for the metaWRAP software. Given complex workflows like metaWRAP it makes sense to streamline operations by using workflow managers such as Snakemake. The authors provide a good overview of the feature of their software, but the addition of more details in different parts of the manuscript might help readers and potential users to get a better picture.

1. The abstract mentions the metaWRAP software, but does not really introduce what metaWRAP does. Although there is clearly a connections to metagenomics, one sentence to introduce the software would make the link more direct.

2. The introduction only focuses on metaWRAP; here, mentioning other, similar tools would make the introduction more inclusive. Why did the authors choose metaWRAP as basis for their software and not another tool?

3. The snakemake-defined rules probably do not require quotes.

4. Yaml should be capitalized to YAML and spelled out when it's first used.

5. The authors state that 100GB RAM and 24 CPUs are sufficient for "normal" jobs. Here, it would be very helpful to give readers and potentials user an idea what "normal" and "larger" jobs correspond to, e.g. number of reads, or libraries?

6. The authors refer to the suitability of the approach for HPC environment. However, are any interfaces to specific scheduling systems included? The most time consuming step of setting up new HPC pipelines usually is the scheduling setup.

7. The authors write that two different datasets completed successfully, but how long did the run take? Of course that's dependent on the hardware used, but it should be quantifiable. 
8. There seems is another, unrelated software on GitHub that uses the same name:

https://github.com/EthanHolleman/metasnake.

Is the rationale for developing the new software tool clearly explained?

Yes

Is the description of the software tool technically sound?

Yes

Are sufficient details of the code, methods and analysis (if applicable) provided to allow replication of the software development and its use by others?

Yes

Is sufficient information provided to allow interpretation of the expected output datasets and any results generated using the tool?

Yes

Are the conclusions about the tool and its performance adequately supported by the findings presented in the article?

Partly

Competing Interests: No competing interests were disclosed.

Reviewer Expertise: Bioinformatics, High Performance Computing, Computational RNA Biology, Cardiovascular Disease

I confirm that I have read this submission and believe that I have an appropriate level of expertise to confirm that it is of an acceptable scientific standard. 
The benefits of publishing with F1000Research:

- Your article is published within days, with no editorial bias

- You can publish traditional articles, null/negative results, case reports, data notes and more

- The peer review process is transparent and collaborative

- Your article is indexed in PubMed after passing peer review

- Dedicated customer support at every stage

For pre-submission enquiries, contact research@f1000.com 